\title{
Construction of Ideological and Political Teaching System for College Students Based on Mobile Intelligent Terminal
}

\author{
Xuefei Tian \\ Department of Tourism Management, Liaoning Jianzhu Vocational College, Shenyang, China
}

Keywords: ideological and political teaching system; mobile intelligent terminal; network topology; storage structure

\begin{abstract}
Intelligent mobile terminal provides people with intelligent learning conditions, which not only enhances the interaction and communication between teachers and students, but also changes the learning style of students, and is also deeply affecting the teaching mode of colleges and universities. This new teaching system breaks through the limitation of time and space in ideological and political teaching in colleges and universities, and provides a more convenient and extensive form of learning for the majority of the students. The system designed in this paper also has the function of collaborative learning, which builds a platform for students to actively discuss, so as to fully embody the students' dominant position. At the same time, the system has also creates a platform for teachers and students to communicate with each other, which helps teachers and students to respect and cooperate with each other in ideological and political teaching and complete the ideological and political task together, and effectively enhances the enthusiasm of the students. The ideological and political teaching of intelligent mobile terminals introduced in this paper can be regarded as an extension of teaching, which is of great significance to deal with the practical problems in ideological and political teaching and practice the concept of omnidirectional learning.
\end{abstract}

\section{Introduction}

In recent years, intelligent mobile terminal has been widely used and popularized in all walks of life. Intelligent mobile phone, computer and other terminal systems have gone through the study and life of college students and play an important role. At this stage, the traditional teaching methods are undergoing tremendous changes. The development of intelligent mobile terminals has widened the way of teaching in Colleges and universities, breaking the traditional teaching mode, and making the teaching in Colleges and universities undergo an intelligent transition. The application of mobile terminal technology makes up for the shortcomings of teaching space, time and resources. And this new model is also gradually introduced into the ideological and political teaching in Colleges and universities, through the perfect combination of information space and physical space, providing the learners with high quality learning resources and enriching the learning experience of the learners in order to achieve the expected effect of Ideological and political teaching in Colleges and universities.

Ideological and political teaching activities are usually arranged in the course of time, showing a strong time mobility characteristics. Students can not better understand the theory of thinking and politics in combination with their own feelings. And did not grasp the convergence of knowledge points, many times, ideological and political teaching formalized and arbitrary. When the teaching activities are carried out, the related information is lagging, and the teachers are difficult to integrate the current social hot spot with the students. The objectives and tasks of the education are not clear enough. In addition, the task of teaching is relatively heavy and involves a wide range. In specific teaching, teachers are also difficult to monitor well, lack of interaction between teachers and students, and do not mobilize all students to participate actively. After the end of the course, the assessment and evaluation are also random or lagging. Generally, there is no effective combination of theoretical education and practical activities, so that students can form a coping mentality to participate in Ideological and political education, and the effect of teaching is poor. 
The main goal of Ideological and political teaching is to help students get closer to the real living environment by participating in practice, and then stimulate their enthusiasm for ideological and political learning and improve the skills of analyzing and solving social reality problems. However, when the educators are engaged in the design of the ideological and political teaching, the individual needs of the students are ignored and the attention to the students' learning state is lack, which is loosely managed by management. There is a lack of interaction in the process of Ideological and political teaching. Teachers and students' communication and information lag make students not only do not have good process help and guidance, and then reduce the effect of communication with other students. These factors seriously affect the students' cooperation and creative thinking.

\section{Mobile Intelligent Terminal with Network Topology}

The measurement of network topology is the foundation of locating and finding faults quickly. Network topology measurement can monitor network topology changes in real time, including link information and node information changes. The global observation ability of SDN enables the controller to make the best decision based on the global network information. In SDN network, network topology discovery is realized through Link Layer Discovery Protocol(LLDP) and OpenFlow message.

LLDP is a two layer protocol that has nothing to do with the manufacturer. It provides a standard link layer discovery mode. It allows network devices to notify their device identities and performance in the local subnet. When other devices receive information, the form of MIB is recalled. Secondly, the LLDP protocol only provides information about the network topology and management configuration, which is not a configuration, control protocol and can not be configured for remote devices through the protocol. The controller sends a LLDP packet through a Packet_out message to a connected switch that sends a LLDP packet to all of its own ports when the switch receives the message. If a switch receives LLDP packets, the link information between the two switches will be sent to the controller through the Packet-in message. The controller can construct the network topology according to these information. Network topology diagram is shown in Figure 1.

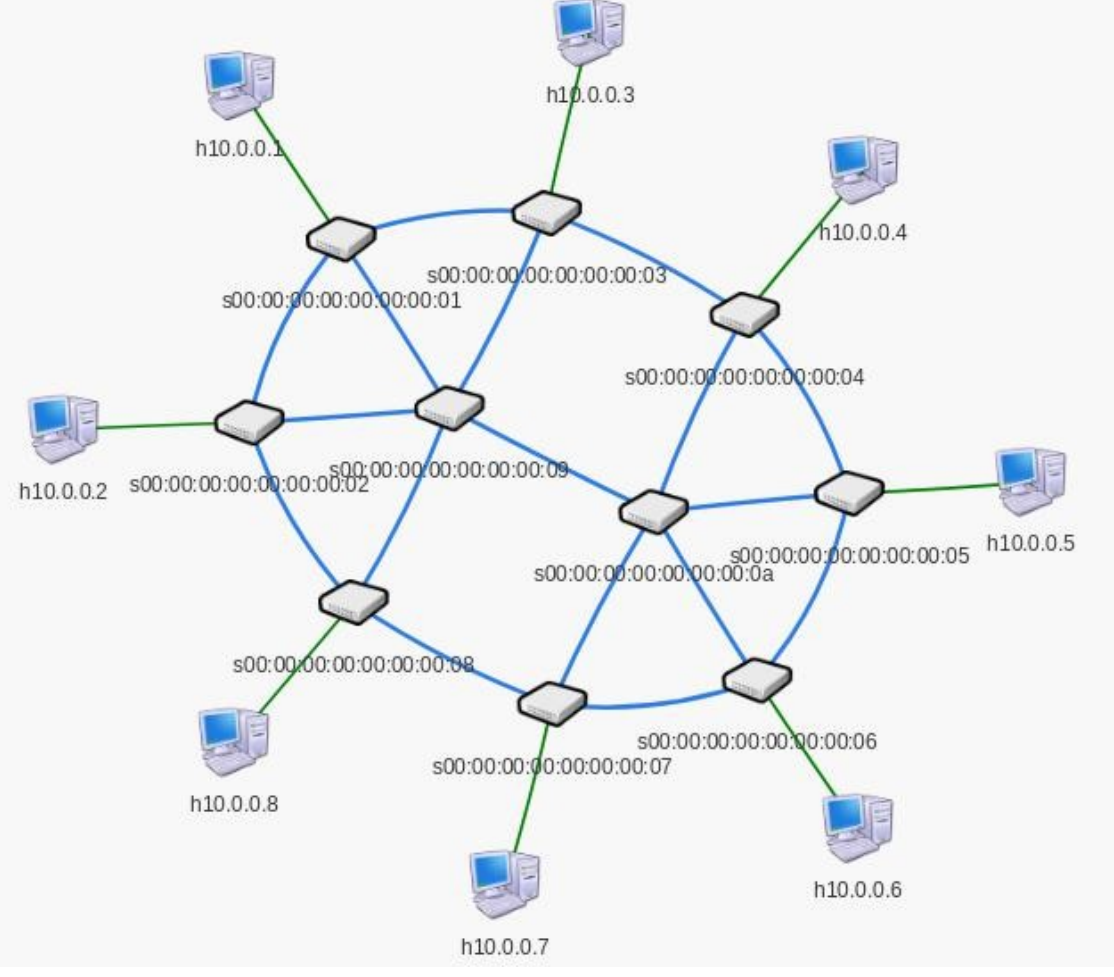

Figure 1. Network topology diagram

The way the network topology is stored is shown in Table 1. 
Table 1 The network topology storage structure table

\begin{tabular}{|l|l|}
\hline key & \multicolumn{1}{|c|}{ value } \\
\hline S1 & S1-S2,S1-S3,S1-S9 \\
\hline S2 & S2-S1,S2-S8,S2-S9 \\
\hline S3 & S3-S1,S3-S4,S3-S9 \\
\hline S4 & S5-S4,S5-S6,S5-Sa \\
\hline S5 & S6-S5,S6-S7,S6-Sa \\
\hline S6 & S7-S6,S7-S8,S7-Sa \\
\hline S7 & S8-S2,S8-S7,S8-S9 \\
\hline S8 & S9-S1,S9-S2,S9-S3,S9-S8,S9-Sa \\
\hline S9 & Sa-S4,Sa-S5,Sa-S6,Sa-S7,Sa-S9 \\
\hline Sa
\end{tabular}

The API provided by Floodlight can get all link and node information and restore the data. The storage mode of network topology determines the speed and efficiency of fault finding. In order to facilitate the quick search of the fault node. This paper designs a topology storage method based on Hash structure, which stores all links of the network topology in the way of key value pairs. Each link is stored in the form of key-value.

\section{Design and Construction of Ideological and Political Teaching System in Colleges and Universities Based on Intelligent Mobile Terminals}

The College Ideological and political teaching system based on intelligent mobile terminal can make full use of the network technology to construct. The school teachers, students and managers use the media of intelligent mobile terminal to carry on the related ideological and political teaching, and together form the main body of the ideological and political teaching. Students use wireless network to learn through mobile terminal related equipment; teachers and managers make use of network and terminal equipment to work out the main tasks of teaching, monitor students' learning process, analyze and summarize the feedback information, and then complete the task of teaching. Because of the different needs of the main body, the specific positioning in the teaching process will also be different. Students are the main subjects of learning. All the functions of the ideological and political teaching activities based on intelligent mobile terminals should be the fundamental goal of designing students' autonomous planning, independent learning and independent evaluation. Teachers and administrators are in a supportive position, playing the role of guidance and assistance. Teachers make use of the teaching plan to provide students with relevant teaching resources. Managers analyze the relevant information of teaching activities, grasp the actual learning situation of students and the teaching process of teachers. In this way, teachers, students and managers in Colleges and universities seem to be in their own space, however, they have an important connection in the teaching of thought and politics. Teachers should grasp the actual needs of the students and then carry out the design of teaching activities and provide relevant information. Students should also communicate with the teachers in time to improve the correctness and effectiveness of the study. The managers are monitoring the behavior of the students, and on the basis of the analysis of relevant information, they are the teachers and students. To provide reasonable assessment, in this way, a dynamic cycle structure is formed between the three, and the operation of the ideological and political teaching system based on intelligent mobile terminals is promoted. 


\section{Summary}

In a word, the design and construction of the ideological and political teaching system based on intelligent mobile terminal is beneficial to the organic combination of practical teaching and mobile learning, which is complementary to the traditional ideological and political teaching model in the past. The design procedure of the new ideological and political teaching system fully takes into account the individual differences of college students, and highlights the students' main position, which is beneficial to the promotion of students' enthusiasm. Through the network technology, it builds the functional modules of pre school preparation, information publication, learning resources, learning collaboration, learning support, evaluation feedback and other functional modules. The related teachers, students, and managers are closely linked and formed the unity of mutual communication and mutual influence, which will surely push the teaching mode of college and university into a new step.

\section{References}

[1] Y Yang, ZD Chen. Research on Brand Professional Construction Based on Engineering Education Certification. Education Teaching Forum, Vol.12 (2016), p. 58.

[2] XB Liu, LI Xin. Construction of Integrative Case Teaching System of Mechanical Engineering Specialty under Engineering Certification Education. Value Engineering, Vol.34 (2018), p. 99.

[3] J Zhang, YH Chu. Research on the Construction of Mixed Curriculum of Software Engineering Specialty Based on Engineering Education Certification. Modern Computer, Vol.21 (2017), p. 65.

[4] JM Lou, SD Bao. Construction of Engineering Training Center for Engineering Education Professional Certification. Research \& Exploration in Laboratory, Vol.9 (2013), p. 87.

[5] XD Meng, YX Zhou. Construction of Graduation Requirements for Microelectronics Science and Engineering Based on Engineering Education Certification. Education Teaching Forum, Vol.42 (2018), p. 132. 